\title{
Low-pH Stability of Influenza A Virus Sialidase Contributing to Virus Replication and Pandemic
}

\author{
Tadanobu Takahashi and Takashi Suzuki* \\ Department of Biochemistry, School of Pharmaceutical Sciences, \\ University of Shizuoka; Shizuoka 422-8526, Japan. \\ Received February 6, 2015; accepted March 31, 2015
}

\begin{abstract}
The spike glycoprotein neuraminidase (NA) of influenza A virus (IAV) has sialidase activity that cleaves the terminal sialic acids (viral receptors) from oligosaccharide chains of glycoconjugates. A new antigenicity of viral surface glycoproteins for humans has pandemic potential. We found "low-pH stability of sialidase activity" in NA. The low-pH stability can maintain sialidase activity under acidic conditions of pH 4-5. For human IAVs, NAs of all pandemic viruses were low-pH-stable, whereas those of almost all human seasonal viruses were not. The low-pH stability was dependent on amino acid residues near the active site, the calcium ion-binding site, and the subunit interfaces of the NA homotetramer, suggesting effects of the active site and the homotetramer on structural stability. IAVs with the low-pH-stable NA showed much higher virus replication rates than those of IAVs with low-pH-unstable NA, which was correlated with maintenance of sialidase activity under an endocytic pathway of the viral cell entry mechanism, indicating contribution of low-pH stability to high replication rates of pandemic viruses. The low-pH-stable NA of the 1968 H3N2 pandemic virus was derived from the low-pH-stable NA of H2N2 human seasonal virus, one of two types classified by both low-pH stability in N2 NA and a phylogenetic tree of N2 NA genes. The 2009 H1N1 pandemic virus acquired low-pH-stable NA by two amino acid substitutions at the early stage of the 2009 pandemic. It is thought that low-pH stability contributes to infection spread in a pandemic through enhancement of virus replication.
\end{abstract}

Key words influenza virus; sialidase; low-pH stability; virus replication; pandemic

\section{INTRODUCTION}

Influenza A virus (IAV) is a zoonotic virus that can infect humans, birds, and mammals including horses and pigs. The virus has two major glycoproteins, hemagglutinin (HA) and neuraminidase (NA), on the viral surface. HA binds to sialic acids on the termini of glycoconjugates on the host cell surface as virus receptors. This binding initiates cell entry of viral particles via an endocytic pathway. ${ }^{1)}$ NA shows sialidase activity, which removes the terminal sialic acid from sugar chains of glycoconjugates. It is widely known that NA facilitates release of progeny virus particles from the host cell surface. ${ }^{2,3)}$ NA also suppresses self-aggregation among progeny viruses by removal of sialic acids in $N$-linked glycoconjugates on HA and NA. ${ }^{4}$ Inhibition of sialidase activity can reduce virus replication. Therefore, sialidase activity of NA is a target of anti-influenza $\mathrm{A}$ and $\mathrm{B}$ virus reagents that are clinically used as influenza virus-specific medicines, including zanamivir. ${ }^{5)}$

NA is a type II transmembrane glycoprotein of a homotetramer structure including the active site, calcium ion-binding site, and $N$-glycosylation site. An NA monomer is formed by four partial domains, a cytoplasmic tail, a transmembrane domain, a stalk region, and a globular domain from N-terminal side. Calcium ion is thought to be required for stability of the active site and the homotetramer structure. ${ }^{2,3,6,7)}$ As of 2014, antigenicities of HA and NA have been classified into 18 and 11 subtypes, respectively. In 2009 and 2010, H17N10 and H18N11 viruses were found from a little yellow-shouldered bat and a flat-faced fruit bat, respectively. Proteins encoding these NA genes are called NA-like proteins because of the lack of NA activity. ${ }^{8-10)}$ Absolute sialidase activity is generally higher for an avian virus than for a human virus. ${ }^{11)}$ Binding activity to red blood cells is shown not only in HA but also in NA. The binding activity is stronger for avian virus NA than for human virus NA. ${ }^{12-14)}$ For sialidase substrate specificity in sialic acid species, all human, swine, and avian viruses are more sensitive to $N$-acetylneuraminic acid than to $N$-glycolylneuraminic acid. ${ }^{15)}$ There are $\alpha 2,6$ and $\alpha 2,3$ types for terminal sialic acid linkages to galactose as IAV receptors. Human virus and avian virus preferentially recognize $\alpha 2,6$-linked and $\alpha 2,3$-linked sialic acids, respectively. ${ }^{1)}$ Sialidase activity of all IAV NAs is reactive to both $\alpha 2,6$-linked and $\alpha 2,3$-linked sialic acids. On the other hand, substrate specificities of almost all IAV NAs are highly selective to $\alpha 2,3$-linked sialic acids over $\alpha 2,6$-linked sialic acids. ${ }^{16-19)}$ Although sialidase activities of some human viruses are more reactive for $\alpha 2,6$-linked sialic acids, sialidase activity for $\alpha 2,6$-linked sialic acids in all of these viruses do not exceed that for $\alpha 2,3$-linked sialic acids. ${ }^{15}$ ) Binding specificities of HA are not necessarily similar to substrate specificities of NA. Although the reason remains unknown, NA may have functions other than facilitation of progeny virus release from the cell surface and prevention of self-aggregation among progeny viruses.

Aquatic birds, such as ducks, are believed to be the natural reservoir for IAV, because almost all subtypes have been isolated from aquatic birds. Avian virus usually replicates in the intestinal tract through the digestive tract of aquatic birds, and infected birds are asymptomatic. ${ }^{20,21)}$ On the other hand, human and swine viruses replicate in the respiratory tract of each host, and infected hosts manifest respiratory symptoms. Infection with highly pathogenic H5N1 avian IAV is often sys- 
temic with a high rate of mortality in chickens and mice. ${ }^{22,23)}$ An approximately 20-amino-acid deletion of the stalk region in NA has been detected in almost all highly pathogenic H5N1 avian viruses isolated since 2007. The deletion of the stalk region increases pathogenicity in mice, ${ }^{24,25)}$ but different results have been reported for chickens: no effect ${ }^{24)}$ and increased pathogenicity. ${ }^{25,26)}$ Since the first isolation of highly pathogenic $\mathrm{H} 5 \mathrm{~N} 1$ avian virus from a 3 -year-old boy in Hong Kong in 1997, the virus has caused frequent human infections, especially in South East Asia. The mortality rate in human infections is approximately $60 \%$. Frequent human infections raise concerns about the pandemic potential of highly pathogenic $\mathrm{H} 5 \mathrm{~N} 1$ avian virus.

IAV contains eight-segmented negative-stranded RNA as a viral genome. Every decade, a new subtype of the virus emerges by genetic reassortment of HA and/or NA genes between human virus and avian or animal virus. This drastic change in the viral surface HA and NA antigens, which is called "an antigenic shift," is a major factor causing a pandemic of IAV. In 1968, new H3N2 virus caused a pandemic, called "Hong Kong Flu." When H2N2 human virus and H3 avian virus simultaneously infected pigs, the H2 HA gene of human virus was replaced by the H3 HA gene of avian virus, resulting in occurrence of the 1968 H3N2 pandemic. ${ }^{27-29)}$ Hong Kong Flu caused two million of deaths mainly by viral pneumonia, most of the deaths occurring in young and elderly adults like a normal seasonal influenza virus. ${ }^{29,30)}$ Since the 1968 pandemic, H3N2 seasonal virus has continued to be epidemic.

Since the 20th century, there have been four pandemics including the 1968 Hong Kong Flu. In the spring of 1918, infection with a new H1N1 virus began to spread from Canton in China. This virus, called Spanish Flu, caused twenty to fifty million deaths worldwide, mostly in relatively young people from 18 to 35 years of age. ${ }^{31,32)}$ High virulence and replication rates of Spanish Flu have been suggested to be due to its HA, NA, and viral RNA polymerase subunits (PB1 or PB2). ${ }^{33,34)}$ HA of Spanish Flu induces excess activation of the immune system, called a cytokine storm, which causes great damage to the host respiratory tract. ${ }^{35)}$ Genetic characterization of Spanish Flu has shown that genes of Spanish Flu are close to intermediates between human virus and swine virus and that Spanish Flu is not directly transmitted from avian virus. The amino acid sequence of Spanish Flu NA is similar to that of swine virus NA but also has characteristics of human virus NA and avian virus NA. ${ }^{36)}$ The mechanism by which Spanish Flu occurs has remained unclear.

In February 1957, a pandemic of a new H2N2 virus, called Asian Flu, started from Hunan in China and caused four million deaths mainly by viral pneumonia, most of the deaths occurring in young and elderly adults like a normal seasonal influenza virus. ${ }^{37)}$ The mortality rate was estimated to be similar to that of the 2009 pandemic. ${ }^{29,30,38)}$ Asian Flu is thought to have occurred by genetic introduction of HA, NA, and PB1 genes in avian virus to those in H1N1 human seasonal virus. ${ }^{30)}$ H2N2 human virus disappeared after Hong Kong Flu in 1968 .

Swine IAV is largely classified into two types, classical type (also called North American type or human-like type) and Eurasia type (also called Europe type or avian-like type). The classical type of swine virus has been inherited among pigs in North America for more than 90 years, and it is believed to have originated from Spanish Flu in 1918. The Eurasia type of swine virus originated from host transmission of H1N1 avian virus to pigs in Europe. ${ }^{39)}$ It has been reported that swine virus often infects humans. Swine virus with triple reassortment among genes of human, avian, and swine viruses sporadically infected humans in 2005-2009. ${ }^{40)}$ In 1976, H1N1 swine virus infected about five hundred people in New Jersey of the United States, one of whom died. ${ }^{39-41)}$ This swine virus did not result in a worldwide pandemic. However, in March 2009, H1N1 swine virus was transmitted to humans in Mexico and caused a pandemic, called pandemic (H1N1) 2009. The NA gene and internal matrix protein $(\mathrm{M})$ gene of pandemic (H1N1) 2009 virus were derived from the Eurasia type of swine virus. The other six genes of pandemic (H1N1) 2009 virus were derived from the classical type of swine virus that was close to triple reassortment swine virus among genes of human, avian, and swine viruses after $1998 .^{40,42)}$

We have found low-pH stability of sialidase activity as one of properties of IAV NA. The low-pH stability of NA means maintenance ability of sialidase activity under an acidic condition of $\mathrm{pH} 4-5$. For human viruses, all of the pandemic viruses in 1918, 1957, 1968, and 2009 had the unique ability of low$\mathrm{pH}$ stability, whereas most seasonal viruses have quickly and irreversibly lost sialidase activity under an acidic condition. We have reported that the low-pH stability of viral sialidase activity enhances virus replication and apprears to contribute to pandemic occurrence. Here, we review the low-pH stability of IAV sialidase activity.

\section{LOW-pH STABILITY OF INFLUENZA VIRUS SIALIDASE ACTIVITY}

We have found different low-pH stabilities in sialidase activity of IAV NAs under an acidic condition of $\left.\mathrm{pH} 4-5 .{ }^{43}\right)$ The low-pH stability did not affect optimal $\mathrm{pH}$ of sialidase activity. In human IAVs, almost all seasonal viruses quickly and irreversibly lost sialidase activity after incubation at $37^{\circ} \mathrm{C}$ within $10 \mathrm{~min}$ under the same acidic condition. On the other hand, all of the pandemic viruses in 1918, 1957, 1968, and 2009 possessed low-pH-stable NA, which maintained sialidase activity even after incubation in an acidic condition at $37^{\circ} \mathrm{C}$ for $10 \mathrm{~min} .^{43-45)}$ Although there are two types of 1918 pandemic virus NA, Leu or Phe at position 256 (numbering based on NA of 1918 pandemic virus), ${ }^{36)}$ the amino acid substitution (L256F) had little effect on the low-pH stability of 1918 pandemic virus NA.4) Sialidase activity of all tested swine viruses showed no low-pH stability similar to those of human seasonal viruses. All avian viruses tested, except most of the highly pathogenic H5N1 avian viruses, showed high low-pH-stable sialidase activity of NA, which was not decreased even after incubation at $\mathrm{pH} 4$ for $1 \mathrm{~h}$ at $37^{\circ} \mathrm{C}$. Sialidase activity of almost all of the highly pathogenic $\mathrm{H} 5 \mathrm{~N} 1$ viruses had no low-pH stability like human seasonal viruses, regardless of the hosts from which they were isolated, including humans, chickens, ducks, and wild birds. ${ }^{46)}$ However, some highly pathogenic H5N1 viruses showed low-pH-stable NA. Sialidase activities of NAs of such viruses were moderately decreased under an acidic condition for more than $10 \mathrm{~min}$ in an incubation time-dependent manner, like properties of the low-pH-stable NAs of pandemic viruses. ${ }^{44,46)}$ H1N1 human 
seasonal virus after the 1918 pandemic temporarily disappeared in the 1950s. In 1977, re-emergence of H1N1 human virus was called Russian Flu as one of the pandemic viruses. However, the NA gene of the virus is identical to that of the H1N1 human seasonal virus in the 1950s. In addition, patients with 1977 H1N1 virus were limited to young people less than 25 years old because of acquisition of immunization against 1918-1950s H1N1 human virus in people more than 25 years old. Therefore, in some recent reviews on influenza pandemics, Russian Flu is not regarded as a pandemic virus. ${ }^{30,31)}$ Our results also demonstrated that the 1977 Russian Flu did not possess low-pH-stable NA, which appeared in pandemic viruses. ${ }^{44)}$ In terms of the low-pH stability, the Russian Flu is different from other pandemic viruses, suggesting that the Russian Flu is close to seasonal virus rather than to pandemic virus. H1N1 human seasonal virus after the 1977 Russian Flu continued to be epidemic until the occurrence of pandemic (H1N1) 2009. Regarding pandemic (H1N1) 2009, most of the new H1N1 viruses after late April in 2009 showed low-pHstable NA, but most of the new H1N1 viruses in March and early April in 2009 did not. $^{45)}$ Results of analyses of the 1968 and 2009 pandemics based on the low-pH stability of sialidase activity will be described later.

\section{LOW-pH STABILITY OF SIALIDASE ACTIVITY MAY RESTRICT HOST SPECIES}

Duck IAV is orally inoculated into ducks together with food and replicates in the duck intestine through an acidic condition in the gizzard ( $\mathrm{pH} 3.6-4.5) .{ }^{47)}$ Human virus cannot infect ducks. Duck virus also cannot infect humans. Thus, the viral host is restricted. ${ }^{20)}$ When ducks were orally inoculated with viruses having the low-pH-stable NA gene of human virus A/Singapore/1/1957 (H2N2) or A/Ann Arbor/6/1960 (H2N2) and the other seven genes of duck virus A/duck/Hong Kong/278/1978 (H2N9), these viruses with the low-pH-stable NA could replicate in the duck intestine. Similarly, when ducks were orally inoculated with a virus having the NA gene of A/Korea/426/1968 (H2N2) and the other seven genes of duck virus A/duck/Hong Kong/278/1978, the virus could not replicate in the duck intestine. ${ }^{11)}$ The NA of A/Korea/426/1968 was phylogenetically very close to the low-pH-unstable NA of A/Taiwan/1/1964 (H2N2). ${ }^{48)}$ Although we have never tested the low-pH stability of sialidase activity of A/Korea/426/1968, it is predicted that the NA of A/Korea/426/1968 is low-pHunstable and that the virus with low-pH-unstable NA cannot replicate in the duck intestine through the duck digestive tract. All NAs of avian viruses (other than most of the highly pathogenic $\mathrm{H} 5 \mathrm{~N} 1$ avian viruses) have high low-pH stability of sialidase activity. ${ }^{43,44,46)}$ The low-pH-stable NA may play a significant role in host restriction of ducks, implying that maintenance of virus sialidase activity through the duck digestive tract is necessary for infection and replication in the duck intestine.

4. AMINO ACID RESIDUES OF NA RESPONSIBLE FOR THE LOW-pH STABILITY OF SIALIDASE ACTIVITY

To identify amino acid residues of NA responsible for the low-pH stability of sialidase activity, we analyzed the low-pH stabilities of chimeric NAs genetically combined between the low-pH-stable NA and the low-pH-unstable NA. In N1 NA of 1918 Spanish Flu A/Brevig Mission/1/1918 (H1N1), two amino acid substitutions of Gln to Arg at position 430 and Gly to Asn at position 454 and an insertion of Thr at position 435 caused loss of the low-pH stability (numbering based on NA of A/Brevig Mission/1/1918). Conversely, in the low-pHunstable N1 NA of A/USSR/92/1977 (H1N1), two amino acid substitutions, Arg to Gln at position 430 and Asn to Gly at position 455, and a deletion of Thr at position 435 resulted in the acquisition of low-pH stability (numbering based on NA of A/USSR/92/1977). ${ }^{44)}$ These residues exist on the surface of the NA structure. The residues at positions 430 and 435 are located near the active site, the calcium ion-binding site, and the subunit interfaces of the NA homotetramer. The residue at position 455 is one of the residues constituting the subunit interfaces $^{49)}$ (Fig. 1A).

In N2 NA of 1968 Hong Kong Flu A/Hong Kong/1/1968 (H3N2), two amino acid substitutions of Arg to Lys at position 344 and Phe to Leu at position 466 caused loss of the low-pH stability of sialidase activity. In the low-pH-unstable N2 NA of A/Texas/1968 (H2N2), a single amino acid substitution of Leu to Phe at position 466 was sufficient to confer low-pH stability similar to that of $\mathrm{A} /$ Hong Kong/1/1968 (numbering based on N2 NA of human virus including A/Hong Kong/1/1968 and A/Texas/1968). ${ }^{50)}$ These residues exist on the surface of the NA molecule. The residue at position 344 is located near the active site and the calcium ion-binding site. ${ }^{3,51,52)}$ The residue at position 466 is near the subunit interfaces of the NA homotetramer ${ }^{53-55)}$ (Fig. 1B).

Two types of viruses, which possess the low-pH-stable NA or low-pH-unstable NA of sialidase activity in 2009 pandemic virus (H1N1), were isolated in the period from March to May in 2009. There were only two amino acid differences between the low-pH-stable NA of A/Norway/3858/2009 (H1N1) and the low-pH-unstable NA of A/California/04/2009 (H1N1). In N1 NA of A/California/04/2009 (H1N1), two amino acid substitutions of Val to Ile at position 106 and Asn to Asp at position 248 conferred low-pH stability similar to that of A/ Norway/3858/2009 (H1N1) [numbering based on NA of pandemic (H1N1) 2009 including A/California/04/2009 and A/ Norway/3858/2009]. ${ }^{45)}$ Residues at positions 106 and 248 exist inside and on the surface of the NA molecule, respectively. The inner residue at position 106 is located near the calcium ion-binding site and the subunit interfaces of the NA homotetramer. The surface residue at position 248 is near the active site and the calcium ion-binding site ${ }^{56)}$ (Fig. 1C).

Almost all of the highly pathogenic H5N1 avian IAVs had low-pH-unstable NA, regardless of the hosts they were isolated from, including humans, chickens, ducks, and wild birds, unlike high low-pH-stable NA of general avian viruses. However, some viruses had low-pH-stable NA, similar to pandemic virus NAs. The amino acid sequence of the low-pH-stable NA in A/duck/Guangdong/1/2001 (H5N1) was most closely related to that of the low-pH-unstable NA in A/Hong Kong/213/2003 (H5N1) among the highly pathogenic H5N1 avian viruses tested. We investigated the low-pH stabilities of chimeric NAs combined between the viruses. In N1 NA of A/duck/ Guangdong/1/2001, two amino acid substitutions of Tyr to His at position 155 and Asn to Lys at position 341 caused loss of the low-pH stability. In N1 NA of A/Hong Kong/213/2003, 
A

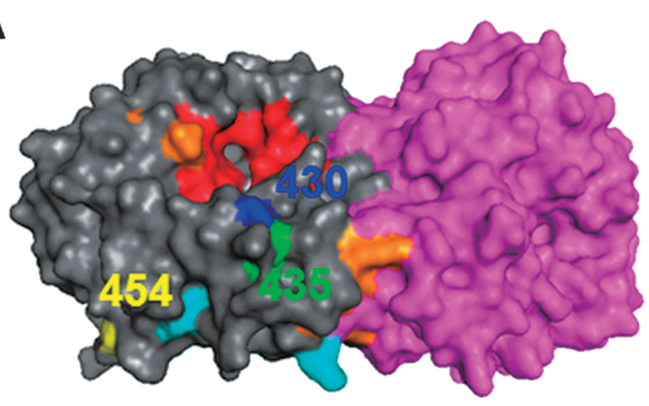

C

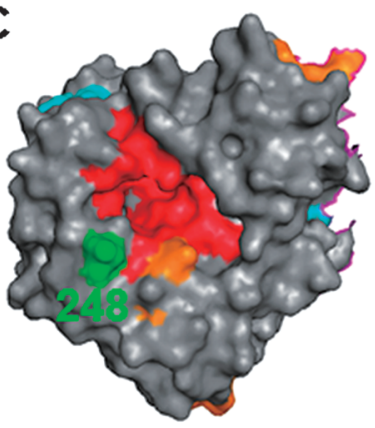

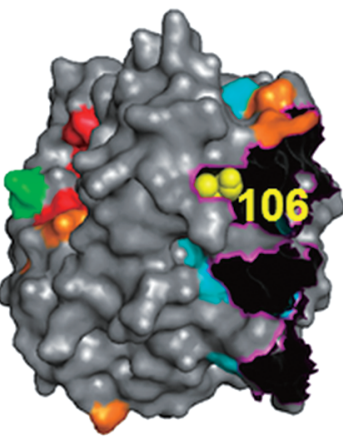

B

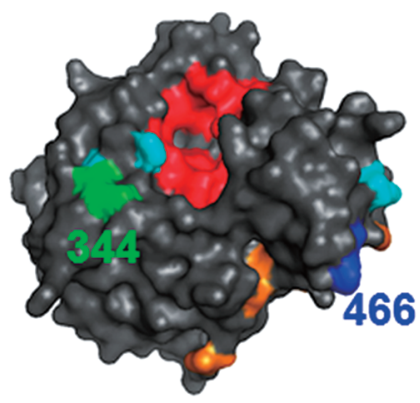

D

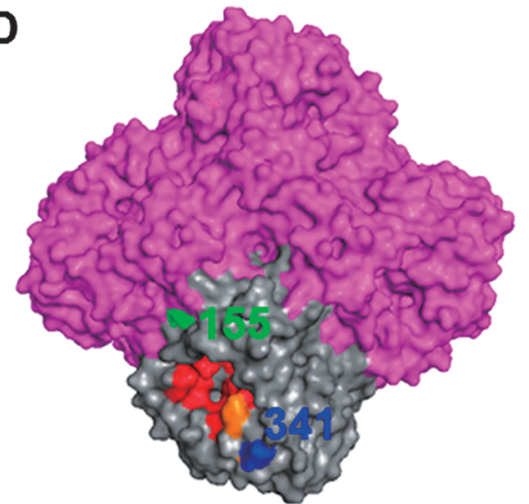

Fig. 1. Location of Amino Acid Residues Responsible for Low-pH Stabilities

Red, orange, and cyan in the figures indicate the active site, calcium-ion binding site, and subunit interfaces, respectively. A surface model of the NA structure was generated by using the Pymol Molecular Graphics System Ver. 1.1r1 (DeLano Scientific LLC). A, Dimer structure of Spanish Flu strain A/Brevig Mission/1/1918 (H1N1) NA (PDB ID, 3BEQ). One subunit of NA is colored in gray. Residues at positions 430, 435, and 454 are colored in blue, green, and yellow, respectively. B, Monomeric structure of A/Tokyo/3/1967 (H2N2) NA (PDB ID, 2BAT) close to NAs of Hong Kong Flu strain A/Hong Kong/1/1968 (H3N2) and A/Texas/1968 (H2N2) at the amino acid sequence level. Residues at positions 344 and 466 are colored in green and blue, respectively. C, Dimer structure of pandemic (H1N1) 2009 strain A/California/04/2009 (H1N1) NA (PDB ID, 3NSS). One subunit of NA is transparent to view the residue at position 106 inside the NA dimer. Residues at positions 106 and 248 are colored in yellow and green, respectively. D, Tetramer structure of highly pathogenic H5N1 avian virus strain A/Viet Nam/1203/2004 (H5N1) NA (PDB ID, 2HTY). One subunit of NA is colored in gray. Residues at positions 155 and 341 are colored in green and blue, respectively.

Table 1. Amino Acid Substitutions Conferring Loss of the Low-pH Stability to the Low-pH-Stable NA

\section{Strain}

1918 pandemic Spanish Flu A/Brevig Mision/1/1918 (H1N1)

1968 pandemic Hong Kong Flu

A/Hong Kong/1/1968 (H3N2)

Pandemic (H1N1) 2009

A/Norway/3858/2009 (H1N1)

Highly pathogenic $\mathrm{H} 5 \mathrm{~N} 1$ avian virus

A/duck/Guangdong/1/2001 (H5N1)
Amino acid substitutions (numbering based on each NA)

Gln430Arg Thr435insertion Gly454Asn

Arg344Lys Phe466Leu

1le106Val Asp248Asn

Tyr155His Asn341Lys

Table 2. Amino Acid Substitutions Conferring Acquisition of the Low-pH Stability to the Low-pH-Unstable NA

\begin{tabular}{cc}
\hline \hline Strain & Amino acid substitutions (numbering based on each NA) \\
\hline H1N1 seasonal human virus & \\
A/USSR/92/1977 (H1N1) & Arg430Gln Thr435deletion Asn455Gly \\
H2N2 seasonal human virus & Leu466Phe \\
A/Texas/1968 (H2N2) & \\
Pandemic (H1N1) 2009 & Val1061le Asn248Asp \\
A/California/04/2009 (H1N1) & His155Tyr Lys341Asn \\
Highly pathogenic H5N1 avian virus & \\
A/Hong Kong/213/2003 (H5N1)
\end{tabular}

the reverse substitutions, His to Tyr at position 155 and Lys to Asn at position 341, resulted in recovery of the low-pH stability to a level similar to that of A/duck/Guangdong/1/2001 (numbering based on NA of A/duck/Guangdong/1/2001 and A/Hong Kong/213/2003). ${ }^{46)}$ These amino acid residues exist on the surface of the NA structure. The residue at position 155 
is located near the subunit interfaces of the NA homotetramer. The residue at position 341 is located near the active site and the calcium ion-binding site ${ }^{57)}$ (Fig. 1D).

Amino acid substitutions conferring loss of low-pH stability to the low-pH-stable NA are summarized in Table 1. Amino acid substitutions conferring acquisition of low-pH stability to the low-pH-unstable NA are summarized in Table 2. All amino acid substitutions affecting the low-pH stability of sialidase activity are located near the active site, the calcium ion-binding site, and/or the subunit interfaces. Calcium ion is thought to be required for stability of the active site and the homotetramer structure. ${ }^{2,3,6,7)}$ Our findings suggest that these substitutions affect the three-dimensional structure of the active site and the homotetramer, resulting in dramatic changes of the low-pH stabilities.

\section{LOW-pH STABILITY OF SIALIDASE ACTIVITY ENHANCES VIRUS REPLICATION}

We have identified the amino acid determinants for loss or acquisition of low-pH stability of virus sialidase activity. Therefore, we generated IAVs with loss or acquisition of low$\mathrm{pH}$ stability by genetic substitutions of the determinants in NA. Firstly, we generated IAVs with the N2 NA gene of A/ Hong Kong/1/1968 or A/Texas/1968 and the other seven genes of A/WSN/1933 (H1N1). The low-pH-stable NA of A/Hong Kong/1/1968 was modified to the low-pH-unstable NA by introduction of two substitutions, Arg344Lys and Phe466Leu. The low-pH-unstable NA of A/Texas/1968 was also modified to the low-pH-stable NA by introduction of a single substitution, Leu466Phe. The two viruses with low-pH-stable NA showed much higher replication rates than those of the corresponding viruses with low-pH-unstable NA. Plaque size of the virus with low-pH-stable NA of A/Hong Kong/1/1968 was larger than that of the virus with low-pH-unstable mutant NA of Arg344Lys and Phe466Leu. A population of dead infected cells is visualized as a plaque when infected cells are cultured in an agarose-containing medium. Large plaque size means high replication ability and/or high cytotoxic ability of the virus. We have also confirmed higher replication ability of IAV with low-pH-stable NA by virus growth curves from measurement of progeny virus titers in culture supernatant. When mice were intranasally inoculated with the viruses (the protocols were pre-approved by the Animal Ethical Committee of the University of Shizuoka), the two viruses with the low-pH-stable NA efficiently replicated in the mouse lungs, but the two viruses with low-pH-unstable NA did not. ${ }^{58)}$

We also generated IAVs with the N1 NA gene of A/ USSR/92/1977 and the other seven genes of A/WSN/1933. The low-pH-unstable NA of A/USSR/92/1977 was modified to the low-pH-stable NA by introduction of two substitutions and one deletion, Arg430Gln, Thr435deletion, and Asn455Gly. The virus with the low-pH-stable mutant NA showed a much higher replication rate than that of the virus with the low-pHunstable NA of A/USSR/92/1977. The results for the N1 NA mutant virus were similar to the results for the virus with the N2 NA gene of A/Hong Kong/1/1968 or A/Texas/1968. Plaque (focus) size of the virus with the low-pH-stable mutant NA of Arg430Gln, Thr435deletion, and Asn455Gly was larger than that of the virus with the low-pH-unstable NA of A/ USSR/92/1977. ${ }^{44}$

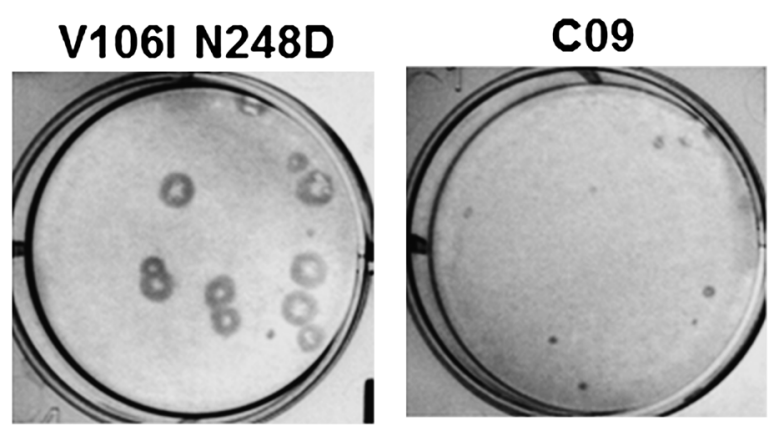

Fig. 2. Focus Formation of IAVs with Low-pH-Stable NA or Low-pHUnstable NA

MDCK cells were infected with A/California/04/2009 (H1N1) with the low-pHunstable NA of A/California/04/2009 (H1N1) (C09) or low-pH-stable mutant NA (V106I N248D). Virus-infected cells were cultured in an agarose-containing medium containing acetylated trypsin for $2 \mathrm{~d}$. Cells were fixed with ethanol-acetic acid $(5: 1, \mathrm{v} / \mathrm{v})$. Plaques were immunostained with rabbit anti-A/WSN/1933 (H1N1) polyclonal antibody. High virus replication forms large foci (immunostained plaques).

We also generated IAVs with the N1 NA gene of pandemic (H1N1) 2009 A/California/04/2009 (H1N1) and the other seven genes of A/California/04/2009. The low-pH-unstable NA of A/California/04/2009 was modified to the low-pHstable NA by introduction of two substitutions, Val106Ile and Asn248Asp, identical to the NA amino acid sequence of $\mathrm{A} /$ Norway/3858/2009 (H1N1). As mentioned in the above results, the virus with the low-pH-stable mutant NA showed a much higher replication rate than that of the virus with the low-pHunstable NA of A/California/04/2009, in both Madin-Darby canine kidney (MDCK) cells (usually used for experiments and replication of IAV) and human lung adenocarcinoma Calu-3 cells. Plaque size of the virus with the low-pH-stable mutant NA of Val106Ile and Asn248Asp was larger than that of the virus with the low-pH-unstable NA of A/California/04/2009 ${ }^{45)}$ (Fig. 2).

Taken together, all viruses with low-pH-stable NA showed much higher replication rates than those of viruses with the low-pH-unstable NA. Viruses with the low-pH-stable NA of A/Hong Kong/1/1968 or the low-pH-stable mutant NA (Leu466Phe) of A/Texas/1968 maintained sialidase activity under an endocytic pathway with the acidic condition being near or below $\mathrm{pH} 5.0 .^{59-61)}$ On the other hand, viruses with the low-pH-unstable NA of A/Texas/1968 or the low-pHunstable mutant NA (Arg344Lys and Phe466Leu) of A/Hong Kong/1/1968 readily lost sialidase activity after initiation of endocytosis. When maintenance of sialidase activity in the low-pH-stable NA under an endocytic pathway was inhibited by an influenza virus sialidase-specific inhibitor, zanamivir, progeny virus production from the virus-infected cells was decreased. ${ }^{58)}$ This result suggests that sialidase activity under an endocytic pathway is important for virus production and replication. Sialidase activity of IAVs is known to be essential for release of progeny virus from the cell surface and prevention of self-aggregation among progeny viruses, at the late stage of the virus lifecycle. Our study raises the possibility of a new significant role of virus sialidase activity in processes at the early stage of virus lifecycle such as virus entry into cells. Some reports have suggested a role of NA at the early stage of the virus lifecycle. A 19-amino-acid deletion in the stalk region of NA increased influenza virus replication in chicken cells but had little effect on release of progeny viruses at the late stage of the virus lifecycle, suggesting that NA plays a 


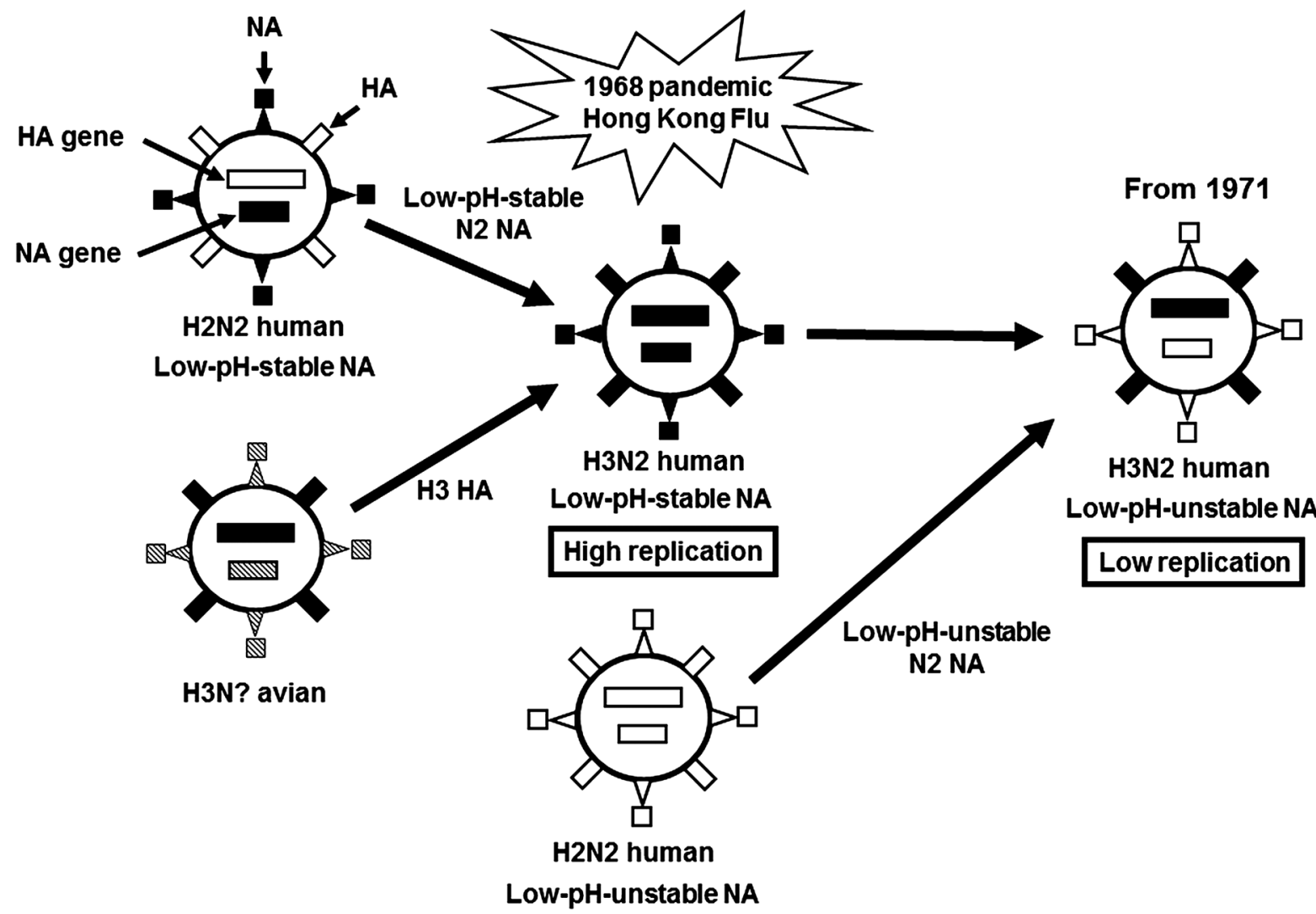

Fig. 3. Transition of NA Genes and Low-pH Stabilities of Sialidase Activities in Occurrence of the 1968 Pandemic Hong Kong Flu

There were two types of H2N2 human virus NAs with low-pH-stable and low-pH-unstable in 1968. When H3N? avian virus and H2N2 human virus simultaneously infected pigs, the H3 HA gene of avian virus was introduced into H2N2 human virus with low-pH-stable NA, resulting in occurrence of a new type of H3N2 human virus, 1968 pandemic Hong Kong Flu. During the 1968 pandemic, probably when H3N2 human virus and H2N2 human virus simultaneously infected humans, the low-pHunstable NA of H2N2 human virus was introduced into Hong Kong Flu by 1971. Loss of the low-pH stability of H3N2 human virus NA currently continues in the present H3N2 human seasonal virus. The virus with low-pH-stable NA showed much higher rate of virus replication than that of the virus with low-pH-unstable NA.

significant role in virus replication at the early stage of the virus lifecycle. ${ }^{26)}$ Glucosyl hesperidin, a derivative of a flavonoid obtained from citrus fruits, suppressed influenza virus infection at the early stage, probably by its inhibitory effect on sialidase activity, although it had no inhibitory effect on the virus receptor binding and no cytotoxicity. ${ }^{62)}$ In the presence of zanamivir, influenza virus at the early stage was deeply embedded in the membrane without inhibitory effects on virus receptor binding (cell surface adsorption) and hemolysis activity (fusion process between the cell membrane and viral membrane) ${ }^{63)}$ When oseltamivir carboxylate (one of the influenza virus sialidase-specific inhibitors) was added at the early stage of cell surface binding and cell entry of influenza virus, virus infection was inhibited. ${ }^{64)}$ Thus, it is likely that sialidase activity of influenza virus has a significant role at the early stage of the virus lifecycle.

\section{ANALYSIS OF PANDEMIC OCCURRENCE IN VIEW OF THE LOW-pH STABILITY OF SIALIDASE ACTIVITY}

Low-pH-stable NAs are presumed to contribute to pandemic occurrence because all pandemic IAVs possess lowpH-stable NA. We analyzed 1968 pandemic Hong Kong Flu in view of the low-pH stability of sialidase activity. In 1968, Hong Kong Flu emerged by genetic introduction of the $\mathrm{H} 3$ HA gene from avian virus to H2N2 human seasonal virus that originated from 1957 Asian Flu. ${ }^{65)}$ At that time, the viral
RNA polymerase subunit PB1 gene of 1968 Hong Kong Flu was reported to be inherited from that of avian virus. $\left.{ }^{66}\right) \mathrm{We}$ found that NAs of H2N2 human seasonal viruses in 1968 could be divided into two types, low-pH-stable NA and low$\mathrm{pH}$-unstable NA types. The two types could also be classified by phylogenetic tree analysis of N2 NA genes. ${ }^{48,67)}$ The lowpH-stable NA of 1968 Hong Kong Flu was inherited from the low-pH-stable NA of H2N2 human seasonal virus, based on both analyses of the phylogenetic tree and the low-pH stability. H3N2 human seasonal virus isolated in 1971 showed no low-pH stability of sialidase activity. The low-pH-unstable NA of H3N2 human seasonal virus in and after 1971 was inherited from the low-pH-unstable NA of H2N2 human seasonal virus in and probably before 1968, based on both analyses of the phylogenetic tree and the low-pH stability ${ }^{67)}$ (Fig. 3). Genetic reassortment between the low-pH-stable NA of 1968 Hong Kong Flu and the low-pH-unstable NA of H2N2 human seasonal virus probably occurred in humans around 1968.

We also analyzed pandemic (H1N1) 2009 in view of the low-pH stability of sialidase activity. Pandemic (H1N1) 2009 emerged by direct transmission of swine virus to humans. The NA gene of pandemic (H1N1) 2009 is derived from that of the Eurasia type of swine virus, which began from direct transmission of H1N1 avian virus to pigs in 1979. All tested swine viruses showed no low-pH stability ${ }^{43)}$ However, it is expected that the NA of pandemic (H1N1) 2009 may easily recover avian virus-like low-pH stability because of NA originated from H1N1 avian virus. Contrary to our expectations, pan- 


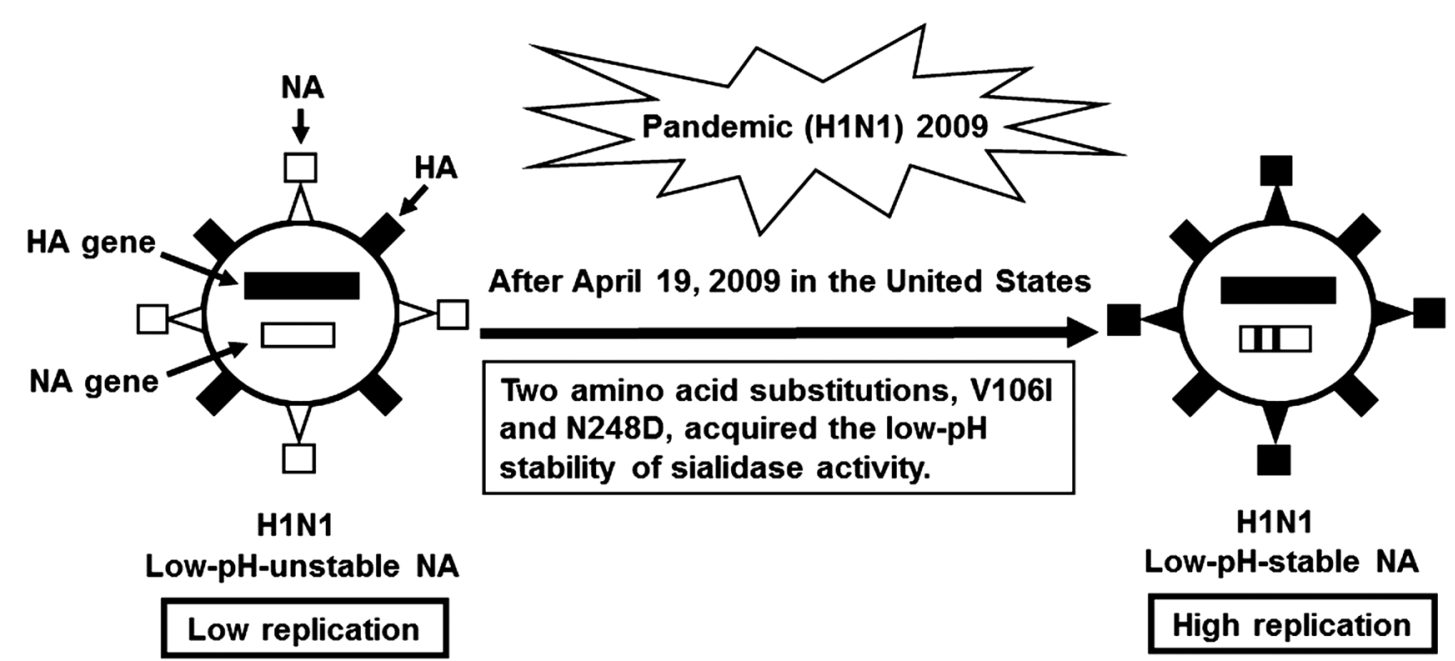

Fig. 4. Acquisition of Low-pH Stability of Sialidase Activity at the Early Stage of the 2009 Pandemic

From March to April in 2009, the new type of H1N1 human virus mainly carried low-pH-unstable NA. After April 19, 2009 in the United States, the H1N1 virus acquired low-pH-stable NA by two amino acid substitutions, Val106Ile and Asn248Asp. Confirmed cases of human infections in the United States also drastically increased after April 19, 2009. This acquisition of low-pH stability of sialidase activity invoked enhancement of virus replication.

demic (H1N1) 2009 viruses had both low-pH-stable and lowpH-unstable NAs. Two amino acid substitutions, Val106Ile and Asn248Asp, were sufficient for acquisition of low-pH stability in the low-pH-unstable NA of 2009 pandemic virus A/California/04/2009. At the early stage of 2009 pandemic (March and April in 2009), pandemic (H1N1) 2009 viruses mainly carried low-pH-unstable NA with Val at position 106 and Asn at position 248. Low-pH-stable NA with Ile at position 106 and Asp at position 248 appeared and drastically spread after April 19, 2009 in the United States ${ }^{45}$ (Fig. 4). In fact, confirmed cases of human infections with pandemic (H1N1) 2009 in the United States drastically increased after April 19, 2009, in terms of date of illness onset (394 patients from March 28 to May 5, 2009). ${ }^{68)}$ This coincidence further supports the speculation that low-pH-stable NA contributed to infection spread in humans during the 2009 pandemic. Hong Kong Flu lost the low-pH stability within 3 years after the 1968 pandemic. Almost all human seasonal viruses have no low-pH stability. The low-pH stability of pandemic (H1N1) 2009 virus will probably be lost in the process of change in the H1N1 human seasonal virus within a few years after 2009 pandemic.

All pandemic viruses possess low-pH-stable NA, which enhances virus replication. The 1918 pandemic Spanish Flu also showed a high virus replication rate in mouse lungs compared with that of H1N1 human seasonal virus. ${ }^{69)}$ Low-pH-stable NA potentially contributes to a pandemic through spread of infection in humans resulting from a high rate of virus replication. On the other hand, almost all human seasonal viruses had loss of low-pH stability after a pandemic. For the 1968 pandemic, our study demonstrated loss of low-pH stability in the H3N2 human seasonal virus isolated from 1971. ${ }^{43,50,67)}$ From a phylogenetic tree of N2 NA genes, A/Taiwan/1/1969 (H3N2) and A/England/878/1969 (H3N2) are predicted to lose low-pH stability by introduction of the low-pH-unstable NA of H2N2 human virus. ${ }^{48)}$ The low-pH-unstable NAs of these H3N2 human viruses is predicted to decrease virus replication compared to the 1968 pandemic virus. This property is maintained in H3N2 human seasonal viruses so far. Why did human seasonal viruses require the loss of low-pH stability (decrease of virus replication)? A pandemic results in immunization of a large number of hosts (humans). A virus absolutely needs a host to replicate and spread infection. Immediately after a pandemic, the large number of immunized hosts would cause a drastic reduction of hosts that influenza virus with the same antigenicity can infect. Therefore, a virus is unable to continue annual repeated infections and replications (epidemic). A pandemic IAV may reduce viral replication and transmission ability by the loss of low-pH stability of sialidase activity for keeping a constant number of non-immunized hosts. At that time, an epidemic can be maintained by an antigen drift of a few amino acid substitutions in viral surface antigens every year. An epidemic is thought to be a stable state to maintain transmissions of a virus among humans for a certain period. One way of coexistence between a virus and a host may be loss of the low-pH stability of sialidase activity by an antigen shift of NA. If we can predict pandemic occurrence in advance, we can prepare for a pandemic or prevent spread of infections in a pandemic. Monitoring of low-pH stability may be useful for selection of pandemic candidates including human, swine, and highly pathogenic H5N1 avian viruses.

Acknowledgments We would like to acknowledge people in our laboratory who contributed to this work. We would also like to acknowledge Dr. Yasuo Suzuki of Chubu University, Dr. Kennedy F. Shortridge of the University of Hong Kong, Dr. Takehiko Saito of the National Institute of Infectious Diseases, Dr. Hideo Goto of the University of Tokyo, Dr. Yoshihiro Kawaoka of the University of Tokyo and the University of Wisconsin-Madison, Dr. Jiasheng Song of the University of Wisconsin-Madison. This work was supported in part by a Grant-in-Aid from the MEXT/JSPS KAKENHI (Scientific Research B, 18390142; Scientific Research C, 23590549; Young Scientist B, 20790357; Challenging Exploratory Research 26670064), CREST (Japan Science and Technology Agency), The Mochida Memorial Foundation for Medical and Pharmaceutical Research, The SRI (Shizuoka Research Institute) academic research grant, Research Foundation for the Electrotechnology of Chubu, and the Global COE Program from the Japan Society for the Promotion of Science. The funders had no role in study design, data collection and analysis, decision 
to publish, or preparation of the manuscript.

Conflict of Interest The authors declare no conflict of interest.

\section{REFERENCES}

1) Suzuki Y. Sialobiology of influenza: molecular mechanism of host range variation of influenza viruses. Biol. Pharm. Bull., 28, 399408 (2005).

2) Colman PM, Varghese JN, Laver WG. Structure of the catalytic and antigenic sites in influenza virus neuraminidase. Nature, 303, 41-44 (1983).

3) Varghese JN, Colman PM. Three-dimensional structure of the neuraminidase of influenza virus $\mathrm{A} / \mathrm{Tokyo} / 3 / 67$ at $2.2 \AA$ resolution. $J$. Mol. Biol., 221, 473-486 (1991).

4) Palese P, Tobita K, Ueda M, Compans RW. Characterization of temperature sensitive influenza virus mutants defective in neuraminidase. Virology, 61, 397-410 (1974).

5) von Itzstein M, Wu WY, Kok GB, Pegg MS, Dyason JC, Jin B, Van Phan T, Smythe ML, White HF, Oliver SW, Colman PM, Varghese JN, Ryan DM, Woods JM, Bethell RC, Hotham VJ, Cameron JM, Penn CR. Rational design of potent sialidase-based inhibitors of influenza virus replication. Nature, 363, 418-423 (1993).

6) Johansson BE, Brett IC. Variation in the divalent cation requirements of influenza a virus N2 neuraminidases. J. Biochem., 134, 345-352 (2003).

7) Brett IC, Johansson BE. Variation in the divalent cation requirements of influenza A virus N1 neuraminidases. J. Biochem., 139, 439-447 (2006).

8) Li Q, Sun X, Li Z, Liu Y, Vavricka CJ, Qi J, Gao GF. Structural and functional characterization of neuraminidase-like molecule N10 derived from bat influenza A virus. Proc. Natl. Acad. Sci. U.S.A., 109, 18897-18902 (2012).

9) Zhu X, Yang H, Guo Z, Yu W, Carney PJ, Li Y, Chen LM, Paulson JC, Donis RO, Tong S, Stevens J, Wilson IA. Crystal structures of two subtype N10 neuraminidase-like proteins from bat influenza A viruses reveal a diverged putative active site. Proc. Natl. Acad. Sci. U.S.A., 109, 18903-18908 (2012).

10) Tong S, Zhu X, Li Y, Shi M, Zhang J, Bourgeois M, Yang H, Chen X, Recuenco S, Gomez J, Chen LM, Johnson A, Tao Y, Dreyfus C, Yu W, McBride R, Carney PJ, Gilbert AT, Chang J, Guo Z, Davis CT, Paulson JC, Stevens J, Rupprecht CE, Holmes EC, Wilson IA, Donis RO. New world bats harbor diverse influenza A viruses. PLoS Pathog., 9, e1003657 (2013).

11) Kobasa D, Wells K, Kawaoka Y. Amino acids responsible for the absolute sialidase activity of the influenza A virus neuraminidase: relationship to growth in the duck intestine. J. Virol., 75, 1177311780 (2001).

12) Hausmann J, Kretzschmar E, Garten W, Klenk HD. N1 neuraminidase of influenza virus A/FPV/Rostock/34 has haemadsorbing activity. J. Gen. Virol., 76, 1719-1728 (1995).

13) Kobasa D, Rodgers ME, Wells K, Kawaoka Y. Neuraminidase hemadsorption activity, conserved in avian influenza A viruses, does not influence viral replication in ducks. J. Virol., 71, 67066713 (1997).

14) Uhlendorff J, Matrosovich T, Klenk HD, Matrosovich M. Functional significance of the hemadsorption activity of influenza virus neuraminidase and its alteration in pandemic viruses. Arch. Virol., 154, 945-957 (2009).

15) Kobasa D, Kodihalli S, Luo M, Castrucci MR, Donatelli I, Suzuki Y, Suzuki T, Kawaoka Y. Amino acid residues contributing to the substrate specificity of the influenza A virus neuraminidase. $J$. Virol., 73, 6743-6751 (1999).

16) Mochalova L, Kurova V, Shtyrya Y, Korchagina E, Gambaryan A, Belyanchikov I, Bovin N. Oligosaccharide specificity of influenza
H1N1 virus neuraminidases. Arch. Virol., 152, 2047-2057 (2007).

17) Mochalova L, Bright R, Xu X, Korchagina E, Chinarev A, Bovin N, Klimov A. Shift in oligosaccharide specificities of hemagglutinin and neuraminidase of influenza B viruses resistant to neuraminidase inhibitors. Glycoconj. J., 27, 321-327 (2010).

18) Li Y, Cao H, Dao N, Luo Z, Yu H, Chen Y, Xing Z, Baumgarth $\mathrm{N}$, Cardona C, Chen X. High-throughput neuraminidase substrate specificity study of human and avian influenza A viruses. Virology, 415, 12-19 (2011).

19) Pourceau G, Chevolot Y, Goudot A, Giroux F, Meyer A, Moulés V, Lina B, Cecioni S, Vidal S, Yu H, Chen X, Ferraris O, Praly JP, Souteyrand E, Vasseur JJ, Morvan F. Measurement of enzymatic activity and specificity of human and avian influenza neuraminidases from whole virus by glycoarray and MALDI-TOF mass spectrometry. ChemBioChem, 12, 2071-2080 (2011).

20) Kida H, Yanagawa R, Matsuoka Y. Duck influenza lacking evidence of disease signs and immune response. Infect. Immun., 30, 547-553 (1980).

21) Ito T, Suzuki Y, Suzuki T, Takada A, Horimoto T, Wells K, Kida $\mathrm{H}$, Otsuki K, Kiso M, Ishida H, Kawaoka Y. Recognition of $\mathrm{N}$ glycolylneuraminic acid linked to galactose by the $\alpha 2,3$ linkage is associated with intestinal replication of influenza A virus in ducks. J. Virol., 74, 9300-9305 (2000).

22) Basler CF, Aguilar PV. Progress in identifying virulence determinants of the $1918 \mathrm{H} 1 \mathrm{~N} 1$ and the Southeast Asian H5N1 influenza A viruses. Antiviral Res., 79, 166-178 (2008).

23) Uchida Y, Chaichoune K, Wiriyarat W, Watanabe C, Hayashi T, Patchimasiri T, Nuansrichay B, Parchariyanon S, Okamatsu M, Tsukamoto K, Takemae N, Ratanakorn P, Yamaguchi S, Saito T. Molecular epidemiological analysis of highly pathogenic avian influenza H5N1 subtype isolated from poultry and wild bird in Thailand. Virus Res., 138, 70-80 (2008).

24) Matsuoka Y, Swayne DE, Thomas C, Rameix-Welti MA, Naffakh N, Warnes C, Altholtz M, Donis R, Subbarao K. Neuraminidase stalk length and additional glycosylation of the hemagglutinin influence the virulence of influenza H5N1 viruses for mice. J. Virol., 83, 4704-4708 (2009).

25) Zhou H, Yu Z, Hu Y, Tu J, Zou W, Peng Y, Zhu J, Li Y, Zhang A, Yu Z, Ye Z, Chen H, Jin M. The special neuraminidase stalk-motif responsible for increased virulence and pathogenesis of $\mathrm{H} 5 \mathrm{~N} 1 \mathrm{influ}-$ enza A virus. PLoS ONE, 4, e6277 (2009).

26) Munier S, Larcher T, Cormier-Aline F, Soubieux D, Su B, Guigand L, Labrosse B, Cherel Y, Quéré P, Marc D, Naffakh N. A genetically engineered waterfowl influenza virus with a deletion in the stalk of the neuraminidase has increased virulence for chickens. $J$. Virol., 84, 940-952 (2010).

27) Kida H, Shortridge KF, Webster RG. Origin of the hemagglutinin gene of H3N2 influenza viruses from pigs in China. Virology, 162, 160-166 (1988).

28) Yasuda J, Shortridge KF, Shimizu Y, Kida H. Molecular evidence for a role of domestic ducks in the introduction of avian $\mathrm{H} 3$ influenza viruses to pigs in southern China, where the A/Hong Kong/68 (H3N2) strain emerged. J. Gen. Virol., 72, 2007-2010 (1991).

29) Li FC, Choi BC, Sly T, Pak AW. Finding the real case-fatality rate of H5N1 avian influenza. J. Epidemiol. Community Health, 62, 555-559 (2008)

30) Oxford JS. Influenza A pandemics of the 20th century with special reference to 1918: virology, pathology and epidemiology. Rev. Med. Virol., 10, 119-133 (2000).

31) Kilbourne ED. Influenza pandemics of the 20th century. Emerg. Infect. Dis., 12, 9-14 (2006).

32) Palese P. Influenza: old and new threats. Nat. Med., 10 (Suppl), S82-S87 (2004).

33) Gottfredsson $\mathrm{M}$, Halldórsson $\mathrm{BV}$, Jónsson $\mathrm{S}$, Kristjánsson $\mathrm{M}$, Kristjánsson $\mathrm{K}$, Kristinsson $\mathrm{KG}$, Löve $\mathrm{A}$, Blöndal $\mathrm{T}$, Viboud $\mathrm{C}$, Thorvaldsson S, Helgason A, Gulcher JR, Stefánsson K, Jónsdóttir 
I. Lessons from the past: familial aggregation analysis of fatal pandemic influenza (Spanish flu) in Iceland in 1918. Proc. Natl. Acad. Sci. U.S.A., 105, 1303-1308 (2008).

34) Pappas C, Aguilar PV, Basler CF, Solórzano A, Zeng H, Perrone LA, Palese P, García-Sastre A, Katz JM, Tumpey TM. Single gene reassortants identify a critical role for PB1, HA, and NA in the high virulence of the 1918 pandemic influenza virus. Proc. Natl. Acad. Sci. U.S.A., 105, 3064-3069 (2008).

35) Kobasa D, Takada A, Shinya K, Hatta M, Halfmann P, Theriault S, Suzuki H, Nishimura H, Mitamura K, Sugaya N, Usui T, Murata T, Maeda Y, Watanabe S, Suresh M, Suzuki T, Suzuki Y, Feldmann $\mathrm{H}$, Kawaoka Y. Enhanced virulence of influenza A viruses with the haemagglutinin of the 1918 pandemic virus. Nature, 431, 703-707 (2004).

36) Reid AH, Fanning TG, Janczewski TA, Taubenberger JK. Characterization of the 1918 "Spanish" influenza virus neuraminidase gene. Proc. Natl. Acad. Sci. U.S.A., 97, 6785-6790 (2000).

37) Van Hoeven N, Pappas C, Belser JA, Maines TR, Zeng H, GarcíaSastre A, Sasisekharan R, Katz JM, Tumpey TM. Human HA and polymerase subunit PB2 proteins confer transmission of an avian influenza virus through the air. Proc. Natl. Acad. Sci. U.S.A., 106, 3366-3371 (2009).

38) Fraser C, Donnelly CA, Cauchemez S, Hanage WP, Van Kerkhove MD, Hollingsworth TD, Griffin J, Baggaley RF, Jenkins HE, Lyons EJ, Jombart T, Hinsley WR, Grassly NC, Balloux F, Ghani AC, Ferguson NM, Rambaut A, Pybus OG, Lopez-Gatell H, AlpucheAranda CM, Chapela IB, Zavala EP, Guevara DM, Checchi F, Garcia E, Hugonnet S, Roth C, The WHO Rapid Pandemic Assessment Collaboration. Pandemic potential of a strain of influenza A (H1N1): early findings. Science, 324, 1557-1561 (2009).

39) Van Reeth K. Avian and swine influenza viruses: our current understanding of the zoonotic risk. Vet. Res., 38, 243-260 (2007).

40) Shinde V, Bridges CB, Uyeki TM, Shu B, Balish A, Xu X, Lindstrom S, Gubareva LV, Deyde V, Garten RJ, Harris M, Gerber S, Vagasky S, Smith F, Pascoe N, Martin K, Dufficy D, Ritger K, Conover C, Quinlisk P, Klimov A, Bresee JS, Finelli L. Triplereassortant swine influenza A (H1) in humans in the United States, 2005-2009. N. Engl. J. Med., 360, 2616-2625 (2009).

41) Robinson JL, Lee BE, Patel J, Bastien N, Grimsrud K, Seal RF, King R, Marshall F, Li Y. Swine influenza (H3N2) infection in a child and possible community transmission, Canada. Emerg. Infect. Dis., 13, 1865-1870 (2007).

42) Garten RJ, Davis CT, Russell CA, Shu B, Lindstrom S, Balish A, Sessions WM, Xu X, Skepner E, Deyde V, Okomo-Adhiambo M, Gubareva L, Barnes J, Smith CB, Emery SL, Hillman MJ, Rivailler P, Smagala J, de Graaf M, Burke DF, Fouchier RA, Pappas C, Alpuche-Aranda CM, López-Gatell H, Olivera H, López I, Myers CA, Faix D, Blair PJ, Yu C, Keene KM, Dotson PD Jr, Boxrud D, Sambol AR, Abid SH, St George K, Bannerman T, Moore AL, Stringer DJ, Blevins P, Demmler-Harrison GJ, Ginsberg M, Kriner P, Waterman S, Smole S, Guevara HF, Belongia EA, Clark PA, Beatrice ST, Donis R, Katz J, Finelli L, Bridges CB, Shaw M, Jernigan DB, Uyeki TM, Smith DJ, Klimov AI, Cox NJ. Antigenic and genetic characteristics of swine-origin 2009A(H1N1) influenza viruses circulating in humans. Science, 325, 197-201 (2009).

43) Takahashi T, Suzuki Y, Nishinaka D, Kawase N, Kobayashi Y, Hidari KI, Miyamoto D, Guo CT, Shortridge KF, Suzuki T. Duck and human pandemic influenza A viruses retain sialidase activity under low pH conditions. J. Biochem., 130, 279-283 (2001).

44) Takahashi T, Kurebayashi Y, Ikeya K, Mizuno T, Fukushima K, Kawamoto H, Kawaoka Y, Suzuki Y, Suzuki T. The low-pH stability discovered in neuraminidase of 1918 pandemic influenza A virus enhances virus replication. PLoS ONE, 5, e15556 (2010).

45) Takahashi T, Song J, Suzuki T, Kawaoka Y. Mutations in NA that induced low $\mathrm{pH}$-stability and enhanced the replication of pandemic (H1N1) 2009 influenza A virus at an early stage of the pandemic.
PLoS ONE, 8, e64439 (2013).

46) Takahashi T, Nidom CA, Quynh Le MT, Suzuki T, Kawaoka Y. Amino acid determinants conferring stable sialidase activity at low $\mathrm{pH}$ for H5N1 influenza A virus neuraminidase. FEBS Open Bio, 2, 261-266 (2012)

47) Webster RG, Yakhno M, Hinshaw VS, Bean WJ, Murti KG. Intestinal influenza: replication and characterization of influenza viruses in ducks. Virology, 84, 268-278 (1978).

48) Lindstrom SE, Cox NJ, Klimov A. Genetic analysis of human H2N2 and early H3N2 influenza viruses, 1957-1972: evidence for genetic divergence and multiple reassortment events. Virology, 328, 101-119 (2004).

49) Xu X, Zhu X, Dwek RA, Stevens J, Wilson IA. Structural characterization of the 1918 influenza virus H1N1 neuraminidase. $J$. Virol., 82, 10493-10501 (2008).

50) Takahashi T, Suzuki T, Hidari KI, Miyamoto D, Suzuki Y. A molecular mechanism for the low-pH stability of sialidase activity of influenza A virus N2 neuraminidases. FEBS Lett., 543, 71-75 (2003).

51) Baker NJ, Gandhi SS. Effect of $\mathrm{Ca}^{2+}$ on the stability of influenza virus neuraminidase. Arch. Virol., 52, 7-18 (1976).

52) Chong AK, Pegg MS, von Itzstein M. Influenza virus sialidase: effect of calcium on steady-state kinetic parameters. Biochim. Biophys. Acta, 1077, 65-71 (1991).

53) Varghese JN, Laver WG, Colman PM. Structure of the influenza virus glycoprotein antigen neuraminidase at $2.9 \AA$ resolution. $\mathrm{Na}$ ture, 303, 35-40 (1983).

54) Varghese JN, Webster RG, Laver WG, Colman PM. Structure of an escape mutant of glycoprotein N2 neuraminidase of influenza virus A/Tokyo/3/67 at $3 \AA$. J. Mol. Biol., 200, 201-203 (1988).

55) Varghese JN, McKimm-Breschkin JL, Caldwell JB, Kortt AA, Colman PM. The structure of the complex between influenza virus neuraminidase and sialic acid, the viral receptor. Proteins, 14, 327-332 (1992).

56) Li Q, Qi J, Zhang W, Vavricka CJ, Shi Y, Wei J, Feng E, Shen J, Chen J, Liu D, He J, Yan J, Liu H, Jiang H, Teng M, Li X, Gao GF. The 2009 pandemic H1N1 neuraminidase N1 lacks the 150-cavity in its active site. Nat. Struct. Mol. Biol., 17, 1266-1268 (2010).

57) Russell RJ, Haire LF, Stevens DJ, Collins PJ, Lin YP, Blackburn GM, Hay AJ, Gamblin SJ, Skehel JJ. The structure of H5N1 avian influenza neuraminidase suggests new opportunities for drug design. Nature, 443, 45-49 (2006).

58) Suzuki T, Takahashi T, Guo CT, Hidari KI, Miyamoto D, Goto $\mathrm{H}$, Kawaoka Y, Suzuki Y. Sialidase activity of influenza A virus in an endocytic pathway enhances viral replication. J. Virol., 79, $11705-11715$ (2005).

59) Overly CC, Lee KD, Berthiaume E, Hollenbeck PJ. Quantitative measurement of intraorganelle $\mathrm{pH}$ in the endosomal-lysosomal pathway in neurons by using ratiometric imaging with pyranine. Proc. Natl. Acad. Sci. U.S.A., 92, 3156-3160 (1995).

60) Patel S, Ramakrishnan L, Rahman T, Hamdoun A, Marchant JS, Taylor CW, Brailoiu E. The endo-lysosomal system as an NAADPsensitive acidic $\mathrm{Ca}^{2+}$ store: role for the two-pore channels. Cell Calcium, 50, 157-167 (2011).

61) Sandhoff K, Harzer K. Gangliosides and gangliosidoses: principles of molecular and metabolic pathogenesis. J. Neurosci., 33, 1019510208 (2013).

62) Saha RK, Takahashi T, Suzuki T. Glucosyl hesperidin prevents influenza a virus replication in vitro by inhibition of viral sialidase. Biol. Pharm. Bull., 32, 1188-1192 (2009).

63) Ohuchi M, Asaoka N, Sakai T, Ohuchi R. Roles of neuraminidase in the initial stage of influenza virus infection. Microbes Infect., $\mathbf{8}$, 1287-1293 (2006).

64) Matrosovich MN, Matrosovich TY, Gray T, Roberts NA, Klenk HD. Neuraminidase is important for the initiation of influenza virus infection in human airway epithelium. J. Virol., 78, 12665-12667 
(2004).

65) Nakao H, Nakajima K, Nakajima S. Location on the evolutionary trees of the non-structural protein (NS) and neuraminidase (NA) genes of late human influenza A (H2N2) viruses: parental viruses of the NS and NA genes of Hong Kong influenza A (H3N2) viruses. J. Gen. Virol., 74, 1667-1672 (1993).

66) Kawaoka Y, Krauss S, Webster RG. Avian-to-human transmission of the PB1 gene of influenza A viruses in the 1957 and 1968 pandemics. J. Virol., 63, 4603-4608 (1989).

67) Suzuki T, Takahashi T, Saito T, Guo CT, Hidari KI, Miyamoto D, Suzuki Y. Evolutional analysis of human influenza A virus N2 neuraminidase genes based on the transition of the low-pH stability of sialidase activity. FEBS Lett., 557, 228-232 (2004).

68) Dawood FS, Jain S, Finelli L, Shaw MW, Lindstrom S, Garten RJ, Gubareva LV, Xu X, Bridges CB, Uyeki TM; Novel Swine-Origin Influenza A (H1N1) Virus Investigation Team. Emergence of a novel swine-origin influenza A (H1N1) virus in humans. N. Engl. J. Med., 360, 2605-2615 (2009).

69) Tumpey TM, Basler CF, Aguilar PV, Zeng H, Solórzano A, Swayne DE, Cox NJ, Katz JM, Taubenberger JK, Palese P, García-Sastre A. Characterization of the reconstructed 1918 Spanish influenza pandemic virus. Science, 310, 77-80 (2005). 